Light-emitting polymers

DOI: $10.1002 / s m l l .200800210$

\section{Sub-50-nm Conjugated Polymer Dots by Nanoprinting**}

\author{
Elisa Mele, Andrea Camposeo, Milena De Giorgi, \\ Francesca Di Benedetto, Carmela De Marco, \\ Vittorianna Tasco, Roberto Cingolani, and \\ Dario Pisignano*
}

Methods for controlling, with high accuracy, the deposition of active molecular materials on surfaces play a fundamental role in the fabrication of functional devices. ${ }^{[1,2]}$ Different patterning techniques have been proposed for organic electronics, including optical methods, ${ }^{[3,4]}$ dip-pen, ${ }^{[5]}$ inkjet, ${ }^{[6]}$ and soft lithography, ${ }^{[7]}$ molecular self-assembly, ${ }^{[8]}$ nanoimprinting, ${ }^{[9]}$ and molding. ${ }^{[10]}$ However, among these approaches, only inkjet and dip-pen lithography have straightforward applications in organic pixel technology. These approaches allow one to realize matrices or arrays of separated features, ${ }^{[5,6]}$ although with limitations in terms of cost and serial throughput. In organic nano- and optoelectronics, a current challenging issue is the research of cheap, parallel-patterning approaches for fabricating i) addressable matrix arrangements of spatially discrete elements, and ii) high-quality emissive features with sub-100-nm size, to be employed as nanoscale light sources. ${ }^{[11]}$ High-resolution soft lithography is in principle able to fulfill these requirements.

In particular, the recently developed particle replication in nonwetting templates (PRINT) ${ }^{[12,13]}$ method was applied to obtain particles using several materials, such as poly(ethylene glycol diacrylate), triacrylate resin, poly(lactic acid), and poly(pyrrole), possibly incorporating bioactive agents. PRINT utilizes a stamp which is not wetted by the target organic materials. If a liquid polymer is placed between a textured and a flat fluorinated surface, the organics tend to be confined within the recessed features of the mold. For this reason, in contrast to other soft lithographic methods, the presence of an

[*] Dr. D. Pisignano, Dr. E. Mele, Dr. A. Camposeo, Dr. M. De Giorgi, Dr. F. Di Benedetto, C. De Marco, Dr. V. Tasco, Prof. R. Cingolani NNL, National Nanotechnology Laboratory of Istituto Nazionale di Fisica della Materia-Consiglio Nazionale delle Ricerche c/o Distretto Tecnologico ISUFI

Via Arnesano, 73100 Lecce (Italy)

E-mail: dario.pisignano@unile.it

Dr. D. Pisignano, C. De Marco

Istituto Superiore di Formazione Interdisciplinare ISUFI Università del Salento

Via Arnesano, 73100 Lecce (Italy)

[**] We acknowledge the financial support from the Regional Strategic Project "Ponamat" and the FIRB Contract RBIP06SH3W, and Dr Alberto Zompatori at Solvay-Solexis for kindly providing the PFPEurethane-methacrylate elastomer. We also thank Stefano Pagliara for optical lithography and Ripalta Stabile for the SEM measurements. D. P. would also like to thank Dr. Sergio Bernardi at Avago Technologies for many helpful discussions. unwanted bottom layer is completely avoided. Moreover, perfluoropolyether (PFPE) derivatives present a series of advantages over standard soft lithography elastomers. ${ }^{[7,14]}$ PFPE compounds allow one to realize features with high resolution and aspect ratios without structural collapses or defects in the transferred pattern. ${ }^{[13]}$ They do not swell in nonpolar solvents, which is a major limitation of poly (dimethylsiloxane), ${ }^{[15]}$ preventing its application in lightemitting conjugated polymers started from liquid solutions. In the field of molecular electronics, conjugated polymers have been successfully employed as active media in display technologies ${ }^{[16]}$ and in solid state lasers ${ }^{[17]}$ by virtue of their high photoluminescence (PL) efficiency over the whole visible spectrum, easy manufacturing, and low cost. Since many conjugated polymers are characterized by good solubility in nonpolar organic solvents (i.e., chloroform and toluene) we believe that PRINT-related methods could open new perspectives for nanopatterned devices and nanoscale light sources based on these materials.

In this work, we report on the realization of isolated lightemitting dots, with lateral dimension down to a few tens of nanometers, by direct PRINT on dissolved conjugated polymers, and on their optical characterization. We employed a photocurable PFPE-urethane methacrylate, different from the methacryloxy-PFPE employed in the pioneering studies on PRINT, ${ }^{[13]}$ but retaining useful properties in terms of high resistance to organic solvents. Emissive microarrays with pixel size $1.5 \mu \mathrm{m}$ and density of about $10^{7} \mathrm{~cm}^{-2}$ were straightforwardly realized. Moreover, we achieved a resolution up to $35 \mathrm{~nm}$, which, to the best of our knowledge, is currently the highest obtained by direct, gentle, top-down patterning on conjugated light-emitting materials. The emission properties of the realized features demonstrate the occurrence of spatial confinement effects in the high-resolution organic semiconductor features, induced by the interaction with the surrounding, oleophobic, fluorinated surfaces. A consequent blueshift of the fluorescence up to $90 \mathrm{meV}$ is observed with respect to reference conjugated-polymer films.

The scheme of the employed lithographic procedure is reported in Figure 1a. As prototype master structures, we fabricated i) a $3 \mu \mathrm{m}$ period array of $1.5 \mu \mathrm{m}$ pitches by photolithography on a silicon substrate, and ii) an array of $\mathrm{In}_{0.5} \mathrm{Ga}_{0.5} \mathrm{As}$ quantum dots, with an average diameter of $36 \mathrm{~nm}$, epitaxially grown on a GaAs substrate by metal organic chemical vapor deposition (MOCVD). The morphology of the masters was faithfully replicated by a PFPE-urethane methacrylate (Solvay Solexis). We also produced untextured PFPE surfaces by depositing and curing the PFPE prepolymer on flat $\mathrm{Si}$ (rms roughness, $r<1 \mathrm{~nm})$, and GaAs substrates $(r \approx 2 \mathrm{~nm})$. The nonswelling behavior of the resulting polymer with nonpolar solvents is crucial for obtaining high quality lightemitting structures. In order to test the general validity of the patterning approach on active materials, we prepared $1.6 \times$ $10^{-5} \mathrm{M}$ chloroform and toluene solutions with several different conjugated polymers: poly[\{2-methoxy-5-(2-ethylhexyloxy $\}$ 1,4-(1-cyanovinylenephenylene))-co-\{2,5-bis( $N, N^{\prime}$-diphenylamino)1,4-phenylene)] (red-emitter, RE), poly[(9,9-dihexylfluorenyl-2, 7-diyl)-alt-co-(9,ethyl-3,6-carbazole)] (blue-emitter), poly[\{9-ethyl-3, 6-bis(2-cyanovinylene)carbazolylene)\}-alt-co-\{2-methoxy-5-(2- 
(a)

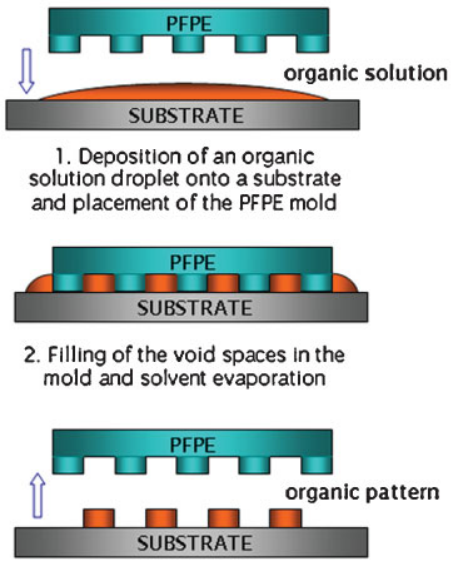

(d)

3. PFPE mold peeling off

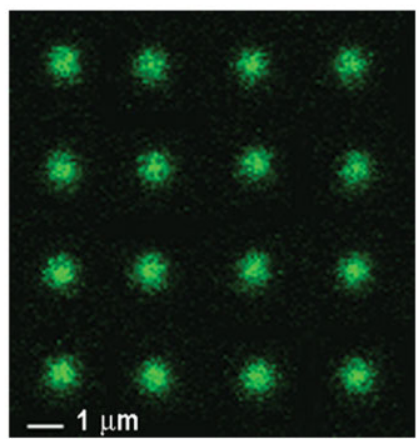

(b)

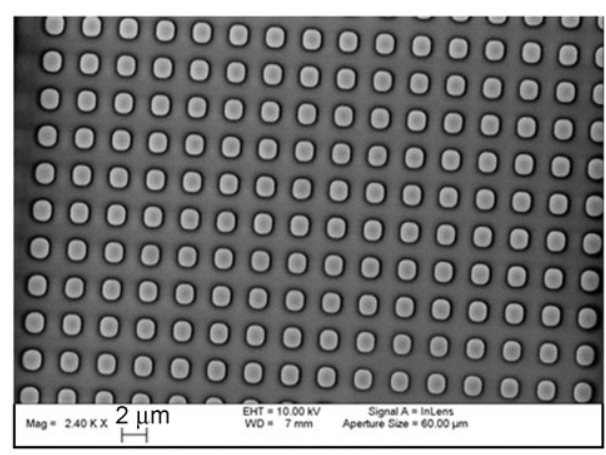

(c)

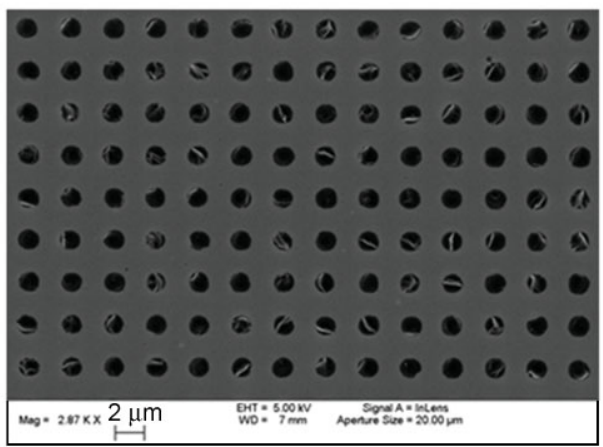

Figure 1. a) Schematic representation of the procedure for high-resolution structuring light-emitting conjugated polymers (features not to scale). In the first phase of the printing process, the relief regions in the replica undergo a conformal contact with the bottom PFPE-derivative substrate, thus forming a reversible sealing in which the conjugated polymer solution is highly confined (in about $2 \mu \mathrm{m}^{3}$ for our microarray samples). The contact between the two printing elements is not driven by the application of an external pressure, propagating instead under the stamp weight (up to $100 \mathrm{mg}$ ) mainly by the spontaneous migration of the conjugated polymer liquid out of the mold or in the recessed replica features. After the complete evaporation of the solvent (1-2 h) at room temperature, the stamp is peeled off, and an array of separated conjugated polymer dots remains onto the previously untextured substrate. SEM images of b) the master, and c) the conjugated polymer microarray. d) Corresponding confocal microscopy of the microarray (period $=3 \mu \mathrm{m}$ ) over an area of about $11 \times 11 \mu \mathrm{m}^{2}$.

ethylhexyloxy)-1,4-phenylene\}] (green-emitter), and poly[9,9dioctylfluorenyl-2,7-diyl)-co-1,4-benzo-\{2,1'-3\}-thiadiazole)] (yellow-emitter). In the following, we will focus on the results with the RE compound, but similar findings hold for all the investigated polymers.

PFPE-based materials generally show low surface energy $\left(14-25 \mathrm{mN} \mathrm{m}^{-1}\right),{ }^{[18]}$ and droplets of conjugated polymer solutions do not wet the elastomer surface, thus reducing their contact area. Most of the liquid solution, therefore, escapes outside the sandwiched area given by the superimposed textured and untextured elastomer elements, a minor volume remaining trapped into the recessed features defined by the patterned stamp. In particular, the non-wetting properties of PFPE-urethane methacrylates by organic-based solutions is functional to obtaining spatially separated features from conjugated-polymer materials. Figure 1(b and c) shows the planar views, imaged by scanning electron microscopy
(SEM), of the master and the lightemitting microarray realized by the RE polymer, with a pixel size and an interpixel distance of about $1.5 \mu \mathrm{m}$. The production of pixels with small diameter, and especially the deposition of the active polymer only in specific, well-separated regions, thus avoiding optical cross-talking among different pixels and background light emission, is particularly important in the microarray field. Indeed, this would determine an increase in the density of active spots in a single light-emitting panel, and a corresponding increase of the storable information. We obtained a pixel density of about $10^{7} \mathrm{~cm}^{-2}$, which is at least three orders of magnitude larger than typically reported microarray values. ${ }^{[19]}$ The high monodispersity and good quality of the fabricated conjugated polymer features are clearly appreciable in Figure 1(c and d), suggesting that the achievable resolution on light-emitting organics can be greatly increased, depending on the starting master. Some limitations to this technology for patterning arrays may arise from the minimum achievable aspect ratios due to the collapse of microstructures, which starts to be observed at a thickness around $100 \mathrm{~nm}$ (for $15-\mu \mathrm{m}$ features). In addition, handling molds for patterning large areas $\left(>\mathrm{cm}^{2}\right)$ can be difficult because of the less favored conformal contact with respect to standard silicone elastomers.

In order to investigate the potential of this method for high-resolution printing on light-emitting conjugated compounds, we carried out soft-lithography experiments on the master quantum dots grown by MOCVD. The size distribution of the quantum dots (Fig. 2a) is well fitted by a Gaussian curve, peaked at $36 \mathrm{~nm}$ with a dispersion (full width at half maximum, FWHM) of about $9 \mathrm{~nm}$ (Fig. 2b). $35 \%, 24 \%$, and $21 \%$ of the dots have a diameter in the range $33-36 \mathrm{~nm}, 30-33 \mathrm{~nm}$, and $36-39 \mathrm{~nm}$, respectively. We first replicated the master morphology on the PFPE-derivative used for obtaining an elastomeric stamp, whose surface morphology is imaged in Figure 2c. The corresponding Gaussian curve, describing the resulting population of recessed nanofeatures, exhibits a peak at $36 \mathrm{~nm}$, with a FWHM of $6 \mathrm{~nm}$ (Fig. 2d). Most of the dots have sizes in the range $33-36 \mathrm{~nm}(34 \%)$, with lower populations in the intervals $30-33 \mathrm{~nm}(23 \%)$, and $36-39 \mathrm{~nm}$ $(16 \%)$. The overall number of replicated dots with sizes varying from 30 to $39 \mathrm{~nm}(73 \%)$ is comparable to the corresponding value for the starting MOCVD master $(80 \%)$. Hence, the stamp reproduces, with excellent fidelity, the negative shape of the master morphology, no heavy loss 
(a)

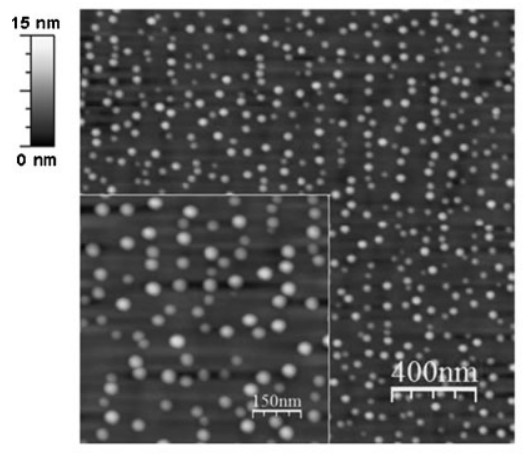

(c)

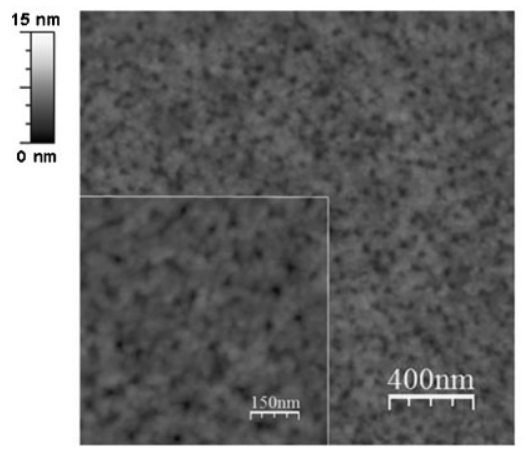

(e)

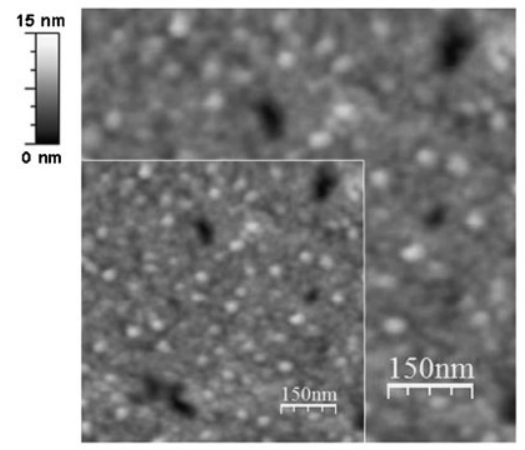

(b)

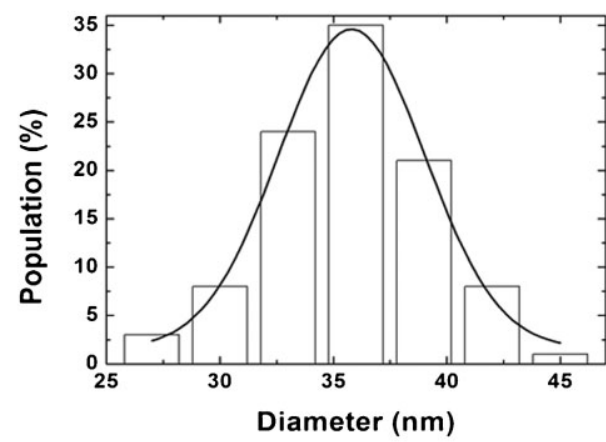

(d)

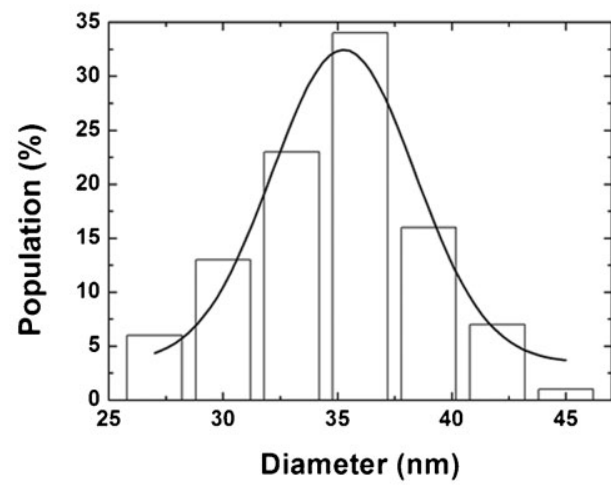

(f)

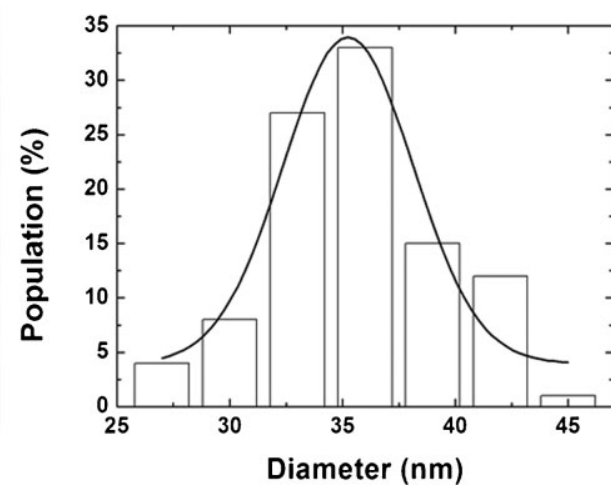

Figure 2. $2 \times 2 \mu \mathrm{m}^{2}$ planar views of sub-50-nm dots, imagined by AFM: a) Si starting master structure, c) PFPE stamp, e) conjugated polymer. Insets: $750 \times 750 \mathrm{~nm}^{2}$ views of the same dots. The corresponding size distributions of dots between 25 and $45 \mathrm{~nm}$ are shown in b) master; d) stamp; f) organics. The superimposed lines are the best fits by Gaussian curves.

being observable either in resolution or in size uniformity upon replication. The stamp was then employed for printing light-emitting conjugated-polymer dots (Fig. 2e), also exhibiting an average diameter of $36 \mathrm{~nm}$, with a width of the distribution in size of $6 \mathrm{~nm}$ (Fig. 2f). In this case, 33\%, 27\%, and $15 \%$ of the particles have diameters between $33-36 \mathrm{~nm}$, $30-33 \mathrm{~nm}$, and $36-39 \mathrm{~nm}$, respectively, with the total number of dots in the range of 30-39 nm (75\%) in very good agreement with the values measured for the master and the stamp. The height of the realized polymer dots measured by atomic force microscopy (AFM) is about $6 \mathrm{~nm}$ (Fig. $3 \mathrm{~b}$ and c). ${ }^{[20]}$
Printing by PFPE-urethane methacrylates is performed entirely at room temperature, and is thus an interesting candidate process for patterning and concomitantly gently preserving the optical functionality of light-emitting organic semiconductors. Moreover, the realization of nanoscale features within conjugated materials can result in a remarkable tailoring of their light-emission spectral properties with interesting spatial confinement effects. ${ }^{[21,22]}$ In order to investigate this issue, we carried out PL and confocal microscopy measurements on the patterned conjugated polymers, comparing the emission properties of sub-50-nm dots and a 200-nmthick film. The PL spectrum of the film was peaked at $668 \mathrm{~nm}$, whereas the PL of an ensemble of polymeric dots (excited through a $1 \mathrm{~mm}$ laser spot) peaked at $650 \mathrm{~nm}$ (Fig. 3a). The microscopy analysis allowed us to investigate this issue in more depth; a typical confocal map of the light-emitting polymeric particles is displayed in Figure 4a, showing appreciable emissive regions corresponding to sample areas with higher local dot densities. The non-uniform dot distribution on the sample surface is attributable to inhomogeneities already present in the masters, and replicated via the PFPE molds. In the regions of higher density, we count more than $3 \times 10^{10}$ dots per $\mathrm{cm}^{2}$, as estimated by the average density value measured with AFM (Fig. 2), and corresponding to a few hundred particles with typical mutual distances of $30-140 \mathrm{~nm}$. In particular, local ensembles of dots composed of about 400-500 light-emitting particles (regions labeled as A and B in Fig. 4a) exhibit PL blueshifts between 15 and $70 \mathrm{meV}$ with respect to the RE film, under identical excitation and detection conditions, and a corresponding decrease of the spectral inhomogeneous broadening (Fig. 4b). Ensembles with about 150 light-emitting particles, such as region $\mathrm{C}$ in Figure 4a, exhibit blueshifts larger than $90 \mathrm{meV}$ with respect to the bulk sample.

The blueshift of the emission spectrum is attributable to spatial confinement effects in the conjugated polymer due to the production of nanofeatures. In contrast to inorganic semiconductors, the optical properties of which are affected by quantum confinement in nanoparticles of size comparable to their exciton Bohr radius, conjugated organic materials generally exhibit much smaller exciton size. In these compounds, the appreciable spatial confinement can instead 
a)

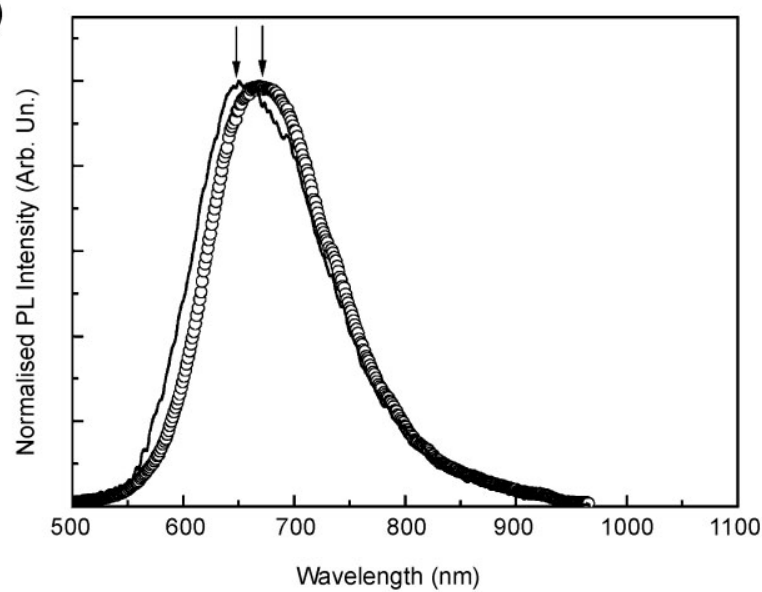

b)

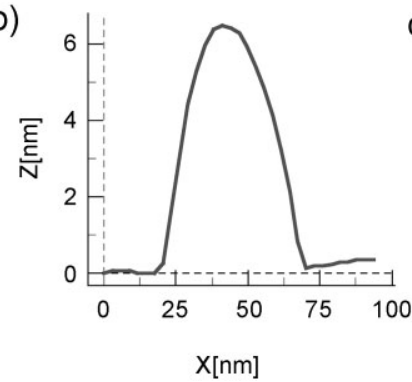

c)

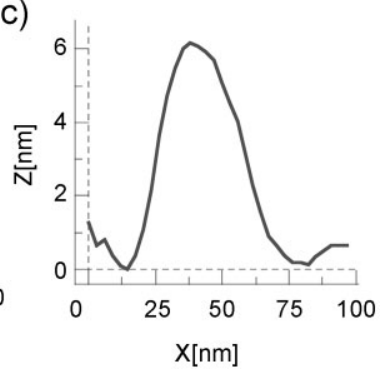

Figure 3. a) Normalized PL spectra of light-emitting polymer film (empty circles), and sub-50-nm dots (solid line). Cross section of a single dot by b) $\ln _{0.5} \mathrm{Ga}_{0.5}$ As (master), and c) RE polymer. (a)

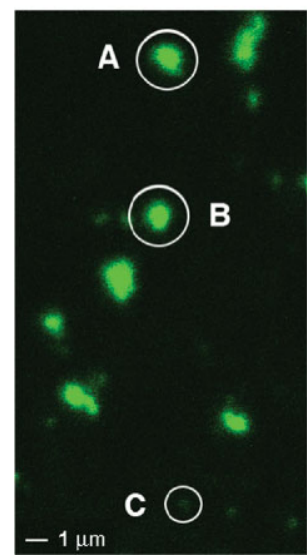

(b)

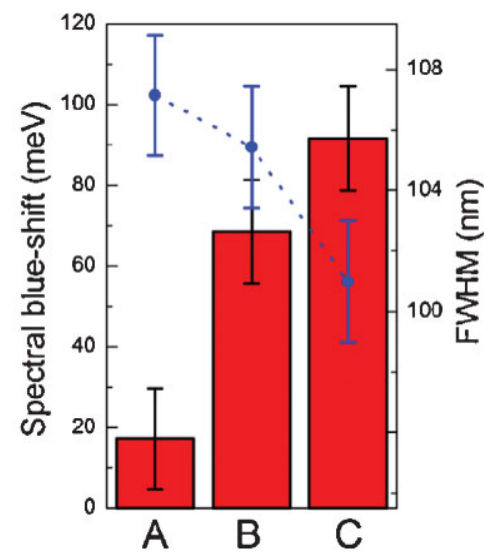

Figure 4. a) RE conjugated-polymer-dot maps imaged by confocal microscopy. The circles evidence areas embedding about 400-500 (circles A and B) and 150 RE particles (circle C), respectively. The appreciable background fluorescence is related to emission from wide substrate regions with lower density of dots. b) Blue-shifts of the regions $A, B$, and $C$, with respect to a reference film (histogram). The corresponding spectral linewidth (FWHM) are shown as circles: $107 \pm 2 \mathrm{~nm}(\mathrm{~A}), 105 \pm 2 \mathrm{~nm}(\mathrm{~B})$, and $101 \pm 2 \mathrm{~nm}$ (C). The FWHM of the reference bulk sample is about $113 \mathrm{~nm}$. be related to breaks along the molecule conjugation lengths, or to the suppression of exciton migration towards chromophore sites of lower energy. The optical transition wavelengths of phenylenevinylene oligomers are expected to increase by 1-2 $\mathrm{nm}$ per bond, extending the conjugation length, ${ }^{[23]}$ and organic nanoparticles consisting of single poly[2-methoxy,5( $2^{\prime}$-ethylhexyloxy)- $p$-phenylenevinylene] (MEH-PPV) chains were found to blueshift by about $30 \mathrm{~nm}$ upon halving the percentage of conjugation double bonds in the molecules. ${ }^{[24]}$ Several works also reported on the influence of film thickness, solution concentration, and nanostructuring on the emission spectra of conjugated polymers, ${ }^{[21,22,25]}$ the photophysical properties of which are remarkably affected by the steric chain conformation of molecules, and by their surrounding micro-/ nanoenvironments, often resulting in PL spectral shifts. ${ }^{[26-32]}$ In particular, the PL peak wavelength for strongly confined or amorphous, random coil conformations of polymer chains tends to decrease with respect to that in bulk samples, resulting instead in more compact packing and consequently more extended $\pi-\pi$ overlaps between different conjugated segments. ${ }^{[26,27,31,33]}$ The blueshift in the PL spectra of our dots with respect to the bulk film is ascribed to a suppression of excitonic migration towards low-energy chromophore sites (i.e., to an increment of the average value of the exciton state energy). ${ }^{[21]}$ This is favored by more amorphous and spatially confined nanoenvironments, and determines stably blueshifted luminescence spectra. ${ }^{[24,34]}$

Smaller dots ensembles, being less affected by the inhomogeneously broadened distribution of emission maxima and by the possible presence of local bulky polymer residues, allow one to appreciate more clearly the spatial confinement effect. Indeed, the isolation of the polymer chains in the nanostructures is forced by the interaction with the surrounding, oleophobic fluorinated surfaces. This will unlikely result in ordered nanoenvironments, thus determining a reduction of the average length of the conjugation segments and of interchain interaction, ${ }^{[25]}$ with a consequent blueshift in the emission. The observed blueshift here is comparable to values previously reported on MEH-PPV single-molecule nanoparticles. ${ }^{[24]}$

In conclusion, we demonstrate PRINT technology for organic optoelectronics, realizing light-emitting microarrays and sub-50-nm features using conjugated polymers. We exploite a PFPE-urethane-methacrylate elastomer, whose nonwetting and high solvent resistant properties are fundamental for manufacturing organic emissive compounds starting with solution-processable materials. As demonstrated in previous works on PRINT, ${ }^{[12]}$ this technology could be used to produce monodisperse colloidal nanoparticles by conjugated polymers upon separation of the top-down fabricated features by the PFPE substrate. Stable aqueous suspensions of fluorescent polymer particles could be obtained ${ }^{[35]}$ by exploiting the limited adhesion of the printed conjugated compound and by substrate sonication. The organic materials still maintain their emission characteristics after the printing procedure, and excitation transfer spatial confinement is observed through the luminescence of sub-50-nm dots. The results indicate PRINT can be applied as an approach for obtaining high spatial control in the deposition of emissive 


\section{communications}

compounds with $10-\mathrm{nm}$-scale resolution, for nanoelectronic and nanophotonic applications.

\section{Experimental Section}

Materials: Poly[\{2-methoxy-5-(2-ethylhexyloxy\}-1,4-(1-cyanovinylenephenylene))-co-\{2,5-bis( $N, N^{\prime}$-diphenylamino)-1,4-phenylene $\left.\}\right]$ (red-emitter, RE), poly[(9,9-dihexylfluorenyl-2,7-diyl)-alt-co(9, ethyl-3,6-carbazole)] (blue-emitter), poly[\{9-ethyl-3,6-bis(2cyanovinylene)carbazolylene)\}-alt-co-\{2-methoxy-5-(2-ethylhexyloxy)1,4-phenylene\}] (green-emitter), and poly[9,9-dioctylfluorenyl-2,7diyl)-co-1,4-benzo- $\left\{2,1^{\prime}-3\right\}$-thiadiazole)] (yellow-emitter) were purchased from American Dye Source, Inc. (Baie D. Urfe, Quebec, Canada) and used as received. Negative tone resist SU-8 2002 for photolithography with a viscosity of around 14000 cSt, SU-8 developer and SU-8 remover PG were purchased from MicroChem (Newton, MA). The PFPE-urethane methacrylate was kindly provided by Solvay Solexis (Italy), and the photoinitiator Darocur 1173 , used for the realization of the PFPE stamps, was obtained from Ciba (Italy). The organic solvents chloroform and toluene, employed for preparing solutions with conjugated polymers, were purchased from J. T. Baker (Phillipsburg, NJ). (100)-oriented GaAs semi-insulating substrates were provided from Wafer Technology LTD. Epichem trimethylgallium and trimethylindium solutions were used together with pure Arsine $\left(\mathrm{AsH}_{3}\right)$ as sources in metalloorganic chemical vapor deposition (MOCVD) growth.

Realization of master structures: The master (i) was fabricated starting from a photoresist pattern generated by optical lithography on Si substrate with EVG620 equipment. A layer of SU-8 2002 (thickness about $1 \mu \mathrm{m}$ ) was deposited onto the surface of the Si substrate by spin-coating $(4000 \mathrm{rpm}$ for $40 \mathrm{~s})$. The resist was subjected to thermal treatment (prebake) at $65^{\circ} \mathrm{C}$ for $1 \mathrm{~min}$ and at $95^{\circ} \mathrm{C}$ for $2 \mathrm{~min}$, in order to guarantee the complete evaporation of the solvent. Then the resist was exposed to UV light $(295-305 \mathrm{~nm})$ for $5.5 \mathrm{~s}$ at a power of $500 \mathrm{~W}$, and thermally cured (post-exposure bake) at $65^{\circ} \mathrm{C}$ for $1 \mathrm{~min}$ and at $95^{\circ} \mathrm{C}$ for $1 \mathrm{~min}$. The pattern was developed in the developer solution, and rinsed by isopropanol. A final hard-bake treatment was carried out at $150^{\circ} \mathrm{C}$ for $5 \mathrm{~min}$. The corresponding photolithographic mask, realized by electron-beam lithography by a Raith 150 system, consisted of a $16 \times 16$ matrix of $1 \mu \mathrm{m} \times 1 \mu \mathrm{m}$ pitches separated by $2 \mu \mathrm{m}$ and by four rulers, $2 \mu \mathrm{m}$ thick. We used a quartz slide (4 inch in diameter), covered by $120 \mathrm{~nm}$-thick $\mathrm{Cr}$ film. A $70 \mathrm{~nm}$ layer of electronic resist (Polymethylmethacrylate 950K) was deposited onto the mask surface by spin-coating at $5500 \mathrm{rpm}$ for $40 \mathrm{~s}$, and thermally cured at $180^{\circ} \mathrm{C}$ for $3 \mathrm{~min}$. The resist was exposed to the electron beam with an exposition dose of $100 \mu \mathrm{Ccm}^{-2}$, and developed for $2 \mathrm{~min}$, and the $\mathrm{Cr}$ layer was finally removed by wet etching for $3 \mathrm{~min}$. The master (ii) was instead realized in a horizontal low pressure-MOCVD system (AIXTRON 200 AIX), equipped with a rotating substrate holder. The reaction chamber pressure was 20 mbar and palladium purified $\mathrm{H}_{2}$ was used as a carrier gas. After the deposition of a $200 \mathrm{~nm}$ undoped GaAs buffer layer, the $\operatorname{In}_{0.5} \mathrm{Ga}_{0.5}$ As quantum dots were formed according to the Stranski-Krastanov growth mode, at a substrate temperature of $550{ }^{\circ} \mathrm{C}$ and with a growth rate of $1 \mathrm{ML} \mathrm{s}^{-1}$.

Realization of PFPE replicas and substrates: For the fabrication of the stamps, we added $4 \% \mathrm{ww}^{-1}$ of the photoinitiator Darocur
1173 to PFPE. The obtained compound was then spin cast at $400 \mathrm{rpm}$ for $40 \mathrm{~s}$ onto the master, and cured by exposition to UV light $(360 \mathrm{~nm})$ under a nitrogen atmosphere. After curing, the PFPE stamp was peeled off from the master. Untextured PFPE surfaces were also produced by depositing and curing the PFPE prepolymer against Si and GaAs surfaces.

Realization of the dots by conjugated polymer solutions: For all the light-emitting polymers, we prepared $1.6 \times 10^{-5} \mathrm{M}$ chloroform and toluene solutions. As reference for the subsequent optical measurements, spin-cast conjugated polymer films were also prepared at this stage from the same solutions. We deposited a droplet (volume $\approx 1 \mu \mathrm{L}$ ) of the polymeric solution onto the untextured PFPE substrate, and the structured replica was then manually positioned on the top of the droplet. The system was stored at room temperature and in an air atmosphere until the evaporation of the solvent was complete (1-2 hours); the stamp was then peeled off.

SEM and AFM characterization: The SEM investigation was performed using the Raith 150 electron beam system operating with an acceleration voltage in the range $5-10 \mathrm{kV}$ and an aperture of $30 \mu \mathrm{m}$. The morphological characterization of the sub-50-nm master consisting of MOCVD-grown InGaAs/GaAs quantum dots, the textured and untextured PFPE replicas, and the final conjugated polymer nanopatterns transferred onto a PFPE substrate was carried out by tapping-mode AFM in air, employing a Nanoscope Illa controller with a Digital Instruments Multimode head integrated with a E-scanner (maximum scan size $15 \times 15 \mu \mathrm{m}^{2}$ ). Silicon tips (Veeco) were employed with a resonant frequency of about $300 \mathrm{kHz}$.

Optical characterization: The fluorescence quantum yield $\left(\Phi_{\mathrm{PL}}\right)$ of the conjugated polymers in the solid state was measured with an integrating sphere, accounting for the excitation light $(390 \mathrm{~nm}$ from a Xe lamp), which is not absorbed by the sample at its first incidence; it is instead absorbed after successive reflections on the reflecting sphere surface. ${ }^{[36]} \Phi_{\mathrm{PL}}$ was found to be $37 \%, 75 \%$, $47 \%$, and $80 \%$, for the red, blue, green, and yellow emitter, respectively. Spatially resolved photoluminescence spectra were collected with a confocal microscope (Olympus FV1000). The samples were excited by a diode laser (Nichia, Japan) emitting at $405 \mathrm{~nm}$ with output power of $60 \mathrm{~mW}$, through a $60 \times$ oil immersion objective lens with a numerical aperture (NA) of 1.40 . The emission from the polymer dots, spatially selected by means of the confocal system, was spectrally analyzed by a $0.32 \mathrm{~m}$ monochromator (iHR320, Jobin Yvon) equipped with a nitrogencooled CCD camera. The spectrometer was directly coupled by means of a lens systems to the confocal head. For the continouswave-photoluminescence measurements, we excited the samples using the diode laser and the forward emission signal from the polymer dots and reference spin-cast films was collected by means of a fiber-coupled monochromator equipped with a CCD detector (Simphony from Jobin Yvon). Typical spectra were collected by employing excitation fluences below $0.5 \mathrm{~mW} \mathrm{~cm}^{-2}$ over spots, using integration times of around $1 \mathrm{~s}$, and averaging over at least ten measurements.

\section{Keywords:}

deposition · light-emitting polymers · nanoprinting · surface modification 
[1] Z. L. Shen, P. E. Burrows, V. Bulovic, S. R. Forrest, M. E. Thompson, Science 1997, 276, 2009.

[2] G. Gustafsson, Y. Cao, G. M. Treacy, F. Klavetter, N. Colaneri, A. J. Heeger, Nature 1992, 357, 477.

[3] a) A. Afzali, C. D. Dimitrakopoulos, T. O. Graham, Adv. Mater. 2003, 15, 2066; b) K. P. Weidkamp, A. Afzali, R. M. Tromp, R. J. Hamers, J. Am. Chem. Soc. 2004, 126, 12740.

[4] a) C. D. Muller, A. Falcou, N. Reckefuss, M. Rojahn, V. Wiederhirn, P. Rudati, H. Frohne, O. Nuyken, H. Becker, K. Meerholz, Nature 2003, 421, 829; b) A. Pogantsch, S. Rentenberger, G. Langer, J. Keplinger, W. Kern, E. Zojer, Adv. Funct. Mater. 2005, 15, 403.

[5] a) B. W. Maynor, S. F. Filocamo, M. W. Grinstaff, J. Liu, J. Am. Chem. Soc. 2002, 124, 522; b) J. H. Lim, C. A. Mirkin, Adv. Mater. 2002, 14, 1474; c) A. Noy, A. E. Miller, J. E. Klare, B. L. Weeks, B. W. Woods, J. J. De Yoreo, Nano Lett. 2002, 2, 109.

[6] a) B. J. De Gans, P. C. Duineveld, U. S. Schubert, Adv. Mater. 2004, 16, 203; b) H. Sirringhaus, T. Kawase, R. H. Friend, T. Shimoda, M. Inbasekaran, W. Wu, E. P. Woo, Science 2000, 290, 2123.

[7] Y. N. Xia, J. A. Rogers, K. E. Paul, G. M. Whitesides, Chem. Rev. 1999, 99, 1823.

[8] D. Philp, J. F. Stoddart, Angew. Chem. Int. Ed. Engl. 1996, 35, 1154.

[9] a) M. Gaal, C. Gadermaier, H. Plank, E. Moderegger, A. Pogantsch, G. Leising, E. J. W. List, Adv. Mater. 2003, 15, 1165; b) E. Mele, F. Di Benedetto, L. Persano, R. Cingolani, D. Pisignano, Nano Lett. 2005, 5, 1915; c) D. Pisignano, L. Persano, P. Visconti, R. Cingolani, G. Gigli, G. Barbarella, L. Favaretto, Appl. Phys. Lett. 2003, 83, 2545; d) E. Mele, A. Camposeo, R. Stabile, P. Del Carro, F. Di Benedetto, L. Persano, R. Cingolani, D. Pisignano, Appl. Phys. Lett. 2006, 89, 131109.

[10] D. Pisignano, L. Persano, R. Cingolani, G. Gigli, F. Babudri, G. M. Farinola, F. Naso, Appl. Phys. Lett. 2004, 84, 1365.

[11] S. P. Price, J. Henzie, T. W. Odom, Small 2007, 3, 372.

[12] J. P. Rolland, B. W. Maynor, L. E. Euliss, A. E. Exner, G. M. Denison, J. M. DeSimone, J. Am. Chem. Soc. 2005, 127, 10096.

[13] J. P. Rolland, E. C. Hagberg, G. M. Denison, K. R. Carter, J. M. DeSimone, Angew. Chem. Int. Ed. 2004, 43, 5796.

[14] Y. Xia, G. M. Whitesides, Angew. Chem. Int. Ed. 1998, 37, 550.

[15] J. N. Lee, C. Park, G. M. Whitesides, Anal. Chem. 2003, 75, 6544.

[16] A. Kraft, A. C. Grimsdale, A. B. Holmes, Angew. Chem. Int. Ed. 1998, 37, 402.

[17] N. Tessler, G. J. Denton, R. H. Friend, Nature 1996, 382, 695.

[18] P. Fabbri, M. Messori, M. Montecchi, S. Nannarone, L. Pasquali, F. Pilati, C. Tonelli, M. Toselli, Polymer 2006, 47, 1055.

[19] I. Barbulovic-Nad, M. Lucente, Y. Sun, M. Zhang, A. R. Wheeler, M. Bussmann, Crit. Rev. Biotechnol. 2006, 26, 237.

[20] Some recessed regions with average dimensions around $150 \mathrm{~nm}$ are observable in the conjugated polymer surface (dark spots in
Fig. 2e), due to the replication of terrace features present in the starting untextured GaAs substrate.

[21] F. Kong, X. L. Wu, G. S. Huang, Y. M. Yang, R. K. Yuan, C. Z. Yang, P. K. Chu, G. G. Siu, J. Appl. Phys. 2005, 98, 074304.

[22] D. Qi, K. Kwong, K. Rademacher, M. O. Wolf, J. F. Young, Nano Lett. 2003, 3, 1265

[23] L. X. Chen, W. J. H. Jager, M. P. Niemczyk, M. R. Wasielewski, J. Phys. Chem. A 1999, 103, 4341.

[24] D. Hu, J. Yu, G. Padmanaban, S. Ramakrishnan, P. F. Barbara, Nano Lett. 2002, 2, 1120.

[25] O. Mirzov, I. G. Scheblykin, Phys. Chem. Chem. Phys. 2006, 8, 5569.

[26] T. Q. Nguyen, R. Y. Yee, B. J. Schwartz, J. Photochem. Photobiol. A 2001, 144, 21.

[27] T. Q. Nguyen, B. J. Schwartz, J. Chem. Phys. 2002, 116, 8198

[28] C. J. Collison, L. J. Rothberg, V. Treemaneekarn, Y. Li, Macromolecules 2001, 34, 2346.

[29] T. Q. Nguyen, V. Doan, B. J. Schwartz, J. Chem. Phys. 1999, 110, 4068.

[30] I. D. W. Samuel, G. Rumbles, C. J. Collison, S. C. Moratti, A. B. Holmes, Chem. Phys. 1998, 227, 75.

[31] M. Zheng, G. L. Bai, D. B. Zhu, J. Photochem. Photobiol. A 1998, 116,143

[32] M. Yan, L. J. Rothberg, E. W. Kwock, T. M. Miller, Phys. Rev. Lett. 1995, 75, 1992.

[33] As an alternative mechanism, the photoinduced incorporation of oxygen upon UV exposure in ambient atmosphere was found to cause conjugation disruption in phenylenevinylene derivatives. (See: M. Atreya, S. Li, E. T. Kang, K. G. Neoh, Z. H. Ma, K. L. Tan, W. Huang, Polym. Degrad. Stab. 1999, 65, 287.) However, this effect, for organic films with thickness of tens of $\mathrm{nm}$, takes place on timescales (tens of minutes) much longer than the integration times employed here.

[34] G. Padmanaban, S. Ramakrishnan, J. Am. Chem. Soc. 2000, 122, 2244.

[35] C. Szymanski, C. Wu, J. Hooper, M. A. Salazar, A. Perdomo, A. Dukes, J. McNeill, J. Phys. Chem. B 2005, 109, 8543.

[36] N. C. Greenham, I. D. W. Samuel, G. R. Hayes, R. T. Phillips, Y. A. R. R. Kessener, S. C. Moratti, A. B. Holmes, R. H. Friend, Chem. Phys. Lett. 1995, 241, 89. 